\title{
Dual Resource Constrained (DRC) Shops: Literature Re- view and Analysis
}

\author{
Matthias Thürer ${ }^{1}$ \\ ${ }^{1}$ Jinan University, Institute of Physical Internet, Zhuhai, PR China \\ matthiasthurereworkloadcontrol.com
}

\begin{abstract}
The majority of manufacturing systems in practice are constrained by machine capacity and labour capacity. Consequently a broad literature on Dual Resource Constrained (DRC) shops exists. However, to best of our knowledge, no systematic review of the literature has been presented. Rather, existing reviews follow an ad-hoc procedure for article selection. In response, this study presents a systematic review of the literature concerned with DRC operating issues, such as worker assignment and production planning and control methods. Results highlight that, while early literature on dual resource constraint job shops was mainly simulation based, recent literature tends towards advanced scheduling mechanisms. This arguably introduces a bias towards deterministic contexts. Moreover, most DRC literature focuses on shop floor control decisions as labour assignment and dispatching. This neglects higher-level planning and control methods, such as order release control.
\end{abstract}

Keywords: Dual Resource Constrained Shop, Systematic Literature Review, Operations Management.

\section{Introduction}

A major aim of job shop research is to align workload and capacity. Most research thereby assumes that capacity is a single variable. However, in practice most manufacturing systems are not only constrained by machine capacity, but also by labour capacity [4]; machines need a worker and workers have to be assigned to the machines. In addition, workers may possess different skills and be able to operate certain machines at different speeds. This type of highly complex shop that is constraint by machines and human resources is known as Dual Resource Constrained (DRC) job shop in the literature.

A broad body of literature on DRC shops exists and, consequently, several reviews have been presented ([25], [12] and [27]). However, existing reviews do not follow a systematic procedure for article retrieval. Rather, a set of papers considered relevant by the authors is discussed. In response, this study presents a systematic review of the literature on DRC shops. The objective is twofold: first, to provide a comprehensive view of the field; second, to update the latest review by [27]. 


\section{Method - Review of the Literature}

This paper starts by asking: What is the current state-of-the-art of the literature on Dual Resource Constrained (DRC) job shops? To answer this question a systematic review of the literature was conducted. The bibliographic database used for sourcing the articles was Scopus - due to its large coverage, e.g. compared to Web of Science, and its accuracy in terms of citation counts, e.g. compared to Google Scholar. In order to keep the number of articles reasonable and to ensure the quality of the sources, the search was restricted to peer-reviewed articles. Scopus was queried in December 2017 using the term: 'Dual Resource Constrained'. To keep results to a manageable number, the search was restricted to the title, abstract, and keywords of papers. There was no restriction on the year of publication or the journals considered (beyond being peer-reviewed). For the search term 82 articles were retrieved. The original sample of 82 articles was further reduced by excluding apparently unrelated articles. Using several channels for retrieving the full articles a total of 43 articles were obtained. Each of these articles was carefully read and analyzed.

\section{$3 \quad$ Results}

[25] subdivided the literature into design issues, such as skill level of workers and associated training, and operating issues, such as worker assignment and production planning and control methods. There is only little research that focuses on the former, mostly focussed on layout decisions. As most previous research, in this study we focus on the operating level. The operating level seeks to align three elements: (i) the workload (or demand), (ii) the machine capacity/capability and (iii) the worker capacity/capability. Most research assumes that the machine capacity/capability is fixed and focuses on the remaining two elements: the workload, which is controlled by dispatching and order release rules, and worker assignment, which is driven by the questions When? Where? and Who?. Another research stream combines the control of the workload and worker assignment by advanced scheduling techniques. However, this presupposes that demand and capacity availability is known in advance and thus deterministic. The following three sections discuss these three aspects.

\subsection{Control of the Workload}

[3] assess the impact of four different job release rules (Immediate Release, Backward Infinite Loading, Forward Finite Loading and Maximum Shop Load) and two dispatching rules (Modified Operation Due Date and Critical Ratio) on a DRC job shop. The authors show that backward Infinite Loading and Forward Finite Loading can improve performance compared to Immediate Release. Both release methods, three levels of labour flexibility (modelled by labour efficiency matrices) and two labour assignment rules were also considered by [23]. Results show no statistically significant performance difference between both release methods. [22] later showed that the relative performance of five dispatching rules was not affected by labour flexibility 
(again modelled by labour efficiency matrices). Finally, the general impact of demand variance was assessed by [9]. The authors show that any reduction of variance through higher planning levels has the potential to dominate performance improvements achievable at order release, dispatching and worker assignment.

\subsection{Worker Assignment}

[8] assess the impact of labour flexibility (this is the number of machines a worker can operate) and compare its impact to staffing decisions, this is a general increase of labour capacity. They highlight that both decisions, staffing and labour flexibility, should play complementary roles. Later [7] assess different levels of flexibility across workers, i.e. some workers being able to work on all machines and some workers only being able to work on one machine. They show that having a small number of highly flexible workers may be a better option than having all workers with average flexibility. However these findings are in the context of perfectly interchangeable workers. A first study to question the assumption of perfectly interchangeable workers was [2] who introduced a labour efficiency matrix that determines the efficiency of a certain worker at a certain machine. [2] investigated five "when" and seven "where" rules demonstrating that a simple "where" rule that moves a worker to the station where the worker is most efficient dominates all other where and when rules. So in addition to deciding when a worker should be transferred and where the worker should be transferred to, managers have to decide whom to transfer. This need to decide who to assign was later emphasized by [5].

There are mainly two types of 'when' rules used in the literature, a 'centralised' rule that transfers a worker each time a job is completed, and a 'decentralised' rule that transfers a worker after completing all jobs (in process and queuing) at the current station. The incorporation of future workload information into the 'when' rules did not improve performance enough to impact manager's decisions [10] On the other hand, there exists a broad set of different 'where' rules. However, the literature typically argues that the 'where' rule has less impact than the 'when' rule [27]. Meanwhile, 'who' rules are dominated by efficiency considerations.

Another important stream in the context of worker assignment, specifically in the context of cross-trained workforces which may possess different skill levels, is learning. [21] assesses the impact of worker learning (in the form of throughput losses that occur during the learning period), worker flexibility (number of stations a worker can operate), and labour attrition (assuming an equal attrition rate across workers). [11] investigate the interplay of learning and attrition (assuming an equal attrition rate across workers), assessing the impact of loosing trained work force and having to train the replacement. This research is extended by [14], which assessed the combined impact of flexibility, staffing and learning (being learning modelled as a decrease in processing with operator task repetition), and [16] and [15] who also include forgetting assessing the interplay of worker flexibility, forgetting rates, attrition rates and flexibility acquisition policies. Using the same learning model [26] later assessed the impact of different part life cycles (i.e. the frequency of changes in produced part 
types). Meanwhile, [28] used a dual-phase learning and forgetting model (DPLFM) to assess the impact of task type on the effectiveness of training and transfer policy.

Finally, a transfer delay was considered by [20] and [24], who assess the interaction of staffing level, distance between stations, buffer size between stations, coefficient of variation and different assignment rules using agent based modelling.

\subsection{Advanced Scheduling Techniques}

The majority of studies discussed in the above two sections assume a stochastic environment. This prohibits the use of scheduling. Rather some greedy heuristics need to be applied. If, on the contrary, the DRC job shop is assumed to be deterministic advanced scheduling techniques can be applied. Different techniques can be identified in the literature. For example: [6] use genetic algorithms to solve the production scheduling problem in DRC job shops; [1] dynamically select worker assignment and dispatching rule employing artificial neural networks (as meta-models to reduce computational complexity) and a fuzzy inference system (to cope with multiple performance criteria); [18] use variable neighbourhood search; [17] use dynamic neighbourhood search in a DRC job shop with interval processing times; [19] use a branch population genetic algorithm; [30] use e knowledge-guided fruit fly optimization algorithm; and, [29] use a hybrid discrete particle swarm optimization. Finally, [13] present a mixed-integer linear programming model to solve the problem of allocating one worker to $n$ tasks (flexibility level) in $m$ cycles within a constrained time period taking into account fatigue and recovery.

\section{Conclusion}

The majority of manufacturing systems in practice are constrained by machine capacity and labour capacity. Consequently a broad literature on Dual Resource Constrained (DRC) shops exists. However, to best of our knowledge, no systematic review of the literature has been presented. In response this paper started by asking: What is the current state-of-the-art of the literature on Dual Resource Constrained (DRC) job shops? The systematic review of the literature highlights that there is a tendency towards advanced scheduling mechanisms in recent years. While early literature on dual resource constraint job shops was mainly simulation based, recent literature tends towards mathematical modelling. However, advanced scheduling mechanisms presuppose a deterministic context. This study therefore calls for more research on DRC shops in a stochastic context as typically for many small and medium sized make-to-order companies that often produce a high variety of order at short notice. Another important observation is the apparent lack of literature on higher-level planning and control methods, such as order release control. Most literature focuses on shop floor control decisions as labour assignment and dispatching. More research is therefore needed that explores the impact of higher level planning and control decisions on DRC performance. 


\section{References}

1. Araz, Ö.U., Salum, L.: A multi-criteria adaptive control scheme based on neural networks and fuzzy inference for DRC manufacturing systems. International Journal of Production Research, 48(1), 251-270 (2010).

2. Bobrowski, P.M., Park, P.S.: An evaluation of labor assignment rules when workers are not perfectly interchangeable. Journal of Operations Management, 11, 257-268 (1993).

3. Bobrowski, P.M., Park, P.S.: Work Release Strategies in a Dual Resource Constrained Job Shop. OMEGA, 17(2), 177-188 (1989).

4. Bokhorst, J.A.C., Gaalman, G.J.C.: Cross-training workers in Dual Resource Constrained systems with heterogeneous processing times. International Journal of Production Research, 47(22), 6333-6356 (2009).

5. Bokhorst, J.C.A., Slomp, J., Gaalman G.J.C.: On the who-rule in Dual Resource Constrained (DRC) manufacturing systems. International Journal of Production Research, 42(23), 5049-5074 (2004)

6. ElMaraghy, H., Patel., V. Abdallah, I.B.: Scheduling of Manufacturing Systems Under Dual-Resource Constraints Using Genetic Algorithms. Journal of Manufacturing Systems, 19(3), 186-201 (2000).

7. Felan, J.T., Fry, T.D.: Multi-level heterogeneous worker flexibility in a Dual Resource Constrained (DRC) job-shop. International Journal of Production Research, 39(14), 30413059 (2001).

8. Felan, J.T., Fry, T.D., Philipoom, P.R.: Labour flexibility and staffing levels in a dualresource constrained job shop. International Journal of Production Research, 31(10), 24872506 (1993).

9. Fredendall, L.D., Melnyk, S.A.: Assessing the impact of reducing demand variance through improved planning on the performance of a dual resource constrained job shop. International Journal of Production Research, 33(6), 1521-1534 (1995).

10. Fredendall, L.D., Melnyk, S.A., Ragatz, G.: Information and scheduling in a dual resource constrained job shop. International Journal of Production Research, 34(10), 2783-2802 (1996).

11. Fry, T.D., Kher, H.V., Malhotra, M.K..: Managing worker flexibility and attrition in dual resource constrained job shops, International Journal of Production Research, 33(8), 21632179 (1995).

12. Hottenstein, M.P., Bowman, S.A.: Cross-training and Worker flexibility: A review of DRC System research. The Journal of High Technology Management Research, 9(2), 157-174 (1998).

13. Jaber, M.Y., Neumann, W.P.: Modelling worker fatigue and recovery in dual-resource constrained systems. Computers \& Industrial Engineering, 59, 75-84 (2010).

14. Kannan, V.R., Jensen, J.B.: Learning and labour assignment in a dual resource constrained cellular shop. International Journal of Production Research, 42(7), 1455-1470 (2004).

15. Kher, H.V.: Examination of flexibility acquisition policies in dual resource constrained job shops with simultaneous worker learning and forgetting effects. Journal of the Operational Research Society, 51, 592-601 (2000).

16. Kher, H.V., Malhotra, M.K., Philipoom, P.R., Fry, T.D.: Modeling simultaneous worker learning and forgetting in dual resource constrained systems. European Journal of Operational Research, 115, 158-172 (1999).

17. Lei, D., Guo, X.: An effective neighbourhood search for scheduling in dual-resource constrained interval job shop with environmental objective. International Journal of Production Economics, 159, 296-303 (2015). 
18. Lei, D., Guo, X.: Variable neighbourhood search for dual-resource constrained flexible job shop scheduling. International Journal of Production Research, 52(9), 2519-2529 (2014).

19. Li, J., Huang, Y., Niu, X.: A branch population genetic algorithm for dual-resource constrained job shop scheduling problem. Computers \& Industrial Engineering, 102, 113-131 (2016).

20. Malhotra, M.K., Kher, H.V.: An evaluation of worker assignment policies in dual resource-constrained job shops with heterogeneous resources and worker transfer delays. International Journal of Production Research, 32(5), 1087-1103 (1994).

21. Malhotra, M.K., Fry, T.D., Kher, H.V., Donohue, J.M.: The Impact of Learning and Labor Attrition on Worker Flexibility in Dual Resource Constrained Job Shops. Decision Sciences, 24(3), 641-664 (1993).

22. Park., S.P.: The examination of worker cross-training in a dual resource constrained job shop. European Journal of Operational Research, 51, 291-299 (1991).

23. Park, S.P., Bobrowski, P.M.: Job Release and Labor Flexibility in a Dual Resource Constrained Job Shop. Journal of Operations Management, 8(3), 230-249 (1989).

24. Sammarco, M., Fruggiero, F., Neumann, W.P., Lambiase, A.: Agent-based modelling of movement rules in DRC systems for volume flexibility: human factors and technical performance. International Journal of Production Research, 52(3), 633-650 (2014).

25. Treleven, M.D.: A Review of the Dual Resource Constrained System Research. IIE Transactions, 21(3), 279-287 (1989).

26. Yue, H., Slomp, J., Molleman, E., Van Der Zee, D.J.: Worker flexibility in a parallel dual resource constrained job shop. International Journal of Production Research, 46(2), 451467 (2008).

27. Xu, J., Xu., X., Xie, S.Q.: Recent developments in Dual Resource Constrained (DRC) system research. European Journal of Operational Research, 215, 309-318 (2011).

28. Zamiska, J.R., Jaber, M.Y., Kher, H.V.: Worker deployment in dual resource constrained systems with a task-type factor. European Journal of Operational Research, 177, 15071519 (2007).

29. Zhang, J., Wang, W., Xu, X.: A hybrid discrete particle swarm optimization for dualresource constrained job shop scheduling with resource flexibility. Journal of Intelligent Manufacturing, 28, 1961-1972 (2017).

30. Zheng, X.L., Wang, L.: A knowledge-guided fruit fly optimization algorithm for dual resource constrained flexible job-shop scheduling problem. International Journal of Production Research, 54(18), 5554-5566 (2016). 